\title{
Europäische Zentralbank: Krisenpolitik ohne Rückendeckung
}

\section{Einleitung ${ }^{1}$}

Mehr als zwei Jahre nach dem Ausbruch der Staatsanleihenkrise im Euroraum ist die Lage weiterhin prekär: Zwar waren die Risikoprämien auf Staatsanleihen der Krisenländer nach der Rettungsaktion der Europäischen Zentralbank (EZB) im Winter 2011/2012 zunächst gefallen, und die Gefahr einer Kreditklemme schien vorerst gebannt, aber seit dem Frühjahr spitzt sich die Lage erneut zu. Die Renditen zehnjähriger Staatsanleihen Portugals, Griechenlands und Irlands sowie Spaniens und Italiens sind weiterhin hoch und belasten infolge der entsprechend geringen Kurse der Wertpapiere die Bilanzen der ohnehin angeschlagenen heimischen Banken (siehe Abbildung 1). ${ }^{2}$ Alle fünf Länder befinden sich zudem in einer Rezession, was ebenfalls die Bankensysteme belastet und zudem das Risiko beinhaltet, dass sich die Krise weiter zuspitzt.

Die sich abzeichnende Rezession in weiten Teilen des Euroraums ist Folge der drastischen Sparmaßnahmen, mit denen die Euroländer - auch auf Drängen der EZB - das Vertrauen der Märkte zurückgewinnen wollen. Im Folgenden wird argumentiert, dass die bisherige Krisenstrategie erhebliche Risiken in sich birgt und bereits hohe Kosten verursacht hat. Das Versagen der Regierungen, die die Krise weder zu Beginn im Keim zu ersticken versuchten noch seither wirksam bekämpften, hatte zur Folge, dass die EZB immer wieder zu Rettungsmaßnahmen gezwungen war, um eine schwere Finanzkrise bzw. das Auseinanderbrechen des Euroraums zu verhindern. Dabei ist die EZB stark in die Kritik geraten, und zwar aus zwei entgegensetzten Richtungen: Während die eine Gruppe von Kritikern argumentiert, die Europäische Zentralbank hätte nicht umfassend genug gehandelt, um der Krise ein Ende zu bereiten, wertet die andere die geldpolitischen Maßnahmen als äußerst risikobehaftet und als eine Überschreitung der Kompetenzen der Währungshüter.

1 Sofern nicht anders gekennzeichnet, stammen die monetären Datenangaben von der EZB, alle anderen aus der AMECO-Datenbank der EU-Kommission bzw. den Datenbanken des World Economic Outlook des Internationalen Währungsfonds. Die Daten über die Renditen der Staatsanleihen stammen von Reuters bzw. Reuters EcoWin.

2 Wenn der Kurs eines Wertpapiers sinkt, steigt die Rendite. Hat eine Staatsanleihe zu Beginn einen Kurswert von 100 Euro und einen Zinssatz von 5 \%, so ist die Rendite 5 Euro. Fällt der Kurswert auf 12,5 Euro, bedeutet die Zinszahlung von 5 Euro aber 40 \% Rendite. Die stark gefallen Kurse belasten die Banken, die diese Wertpapiere halten, da sie Verluste auf der Aktivseite ihrer Bilanz darstellen. Die Banken müssen diese Verluste abschreiben, da Wertpapiere in der Regel nicht zum Ankaufswert, sondern zum Marktwert zu bilanzieren sind. 


\section{Abbildung 1: Renditen zehnjähriger Staatsanleihen ausgewählter Euroländer Tageswerte in Prozent}

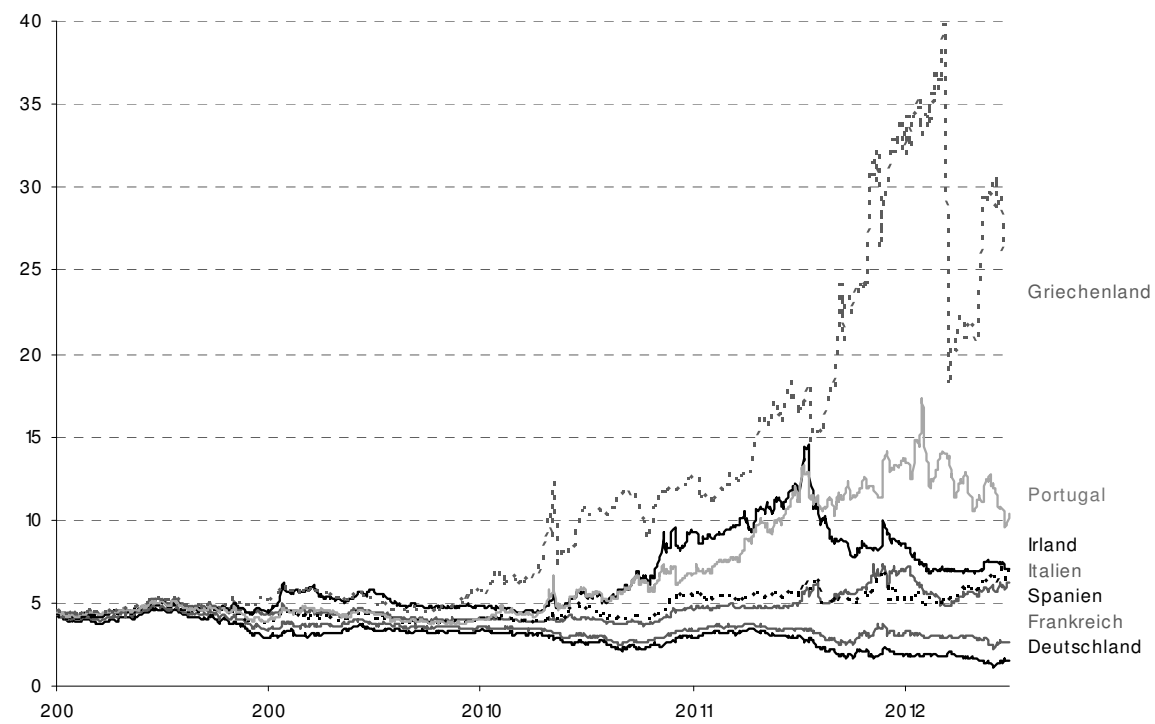

Quelle: Reuters EcoWin (EcoWin Financial).

Tatsächlich birgt die aktuelle Geldpolitik erhebliche Risiken, allerdings nicht, weil die EZB zu weit gegangen ist, sondern weil sie nicht weit genug gegangen ist. Ohne ihre Kompetenzen zu überschreiten, hätte die EZB aber keine darüber hinausgehenden Maßnahmen ergreifen können, die das Vertrauen auf den Märkten hätten wiederherstellen können. Zur Legitimation darüber hinausgehender Maßnahmen bedarf es der Rückendeckung durch die Politik. Nur wenn die Regierungen weitreichende Garantien für die Staatsschuldtitel der Euroländer aussprechen, beispielsweise im Rahmen des vom Sachverständigenrat vorgeschlagenen Schuldentilgungsfonds, ${ }^{3}$ kann die EZB ihrerseits erklären, dass sie zur Stabilisierung der Kurse bzw. Zinsen Staatsanleihen in unbegrenztem Umfang kaufen wird. Allerdings wird auch das für sich genommen mittlerweile nicht mehr ausreichen, um das Vertrauen der Investoren wiederherzustellen. Der ohne Zweifel erforderliche Konsolidierungskurs in den Krisenländern muss zeitlich gestreckt und von einer Wachstumsstrategie begleitet werden, die den Abbau der außenwirtschaftlichen Ungleichgewichte im Euroraum ermöglicht.

3 Sachverständigenrat 2011, S. 4 ff. und 109 ff.

Leviathan, 40. Jg., 3/2012 


\section{Die bisherige Krisenstrategie: Stückwerk}

In den ersten Monaten des Jahres 2010 spitzte sich die Lage am Markt für griechische Staatsanleihen zu. Die Renditen waren bereits seit Oktober 2009 deutlich gestiegen, als der neu gewählte Regierungschef Papandreou erklärt hatte, dass das Defizit der öffentlichen Haushalte von der Vorgänger-Regierung zu niedrig ausgewiesen worden war. Mit nahe 7 \% waren die Renditen für zehnjährige Staatsanleihen Anfang Februar 2010 so hoch, dass Griechenland sein laufendes Budgetdefizit und fällig werdende Staatsschuldtitel nicht hätte finanzieren können. Während Frankreichs Präsident Nicolas Sarkozy und EU-Kommissionspräsident Barroso Griechenland zügig unter die Arme greifen wollten, lehnte Kanzlerin Merkel eine Unterstützung zunächst ab. Ende Februar 2010 erklärte sie im deutschen Fernsehen, Deutschland könne Griechenland dadurch am besten helfen, »dass wir deutlich machen: Griechenland soll seine Hausaufgaben machen « ${ }^{4}$ Ende März stellte sie im Vorfeld des EU-Gipfels in einer Regierungserklärung Notkredite als ultima ratio in Aussicht. Das Defizit der griechischen öffentlichen Haushalte für das Jahr 2009, das Mitte 2009 von der griechischen Regierung noch mit 3,7 \% des BIP geschätzt wurde, wurde im Januar 2010 mit 12,7 \% beziffert, Ende April nach intensiver Prüfung der EU-Kommission abermals revidiert, und zwar auf 13,5\%. Inzwischen liegt es laut IWF bei 15,6 \%. Die zunächst mit $113 \%$ ausgewiesene Staatsschuldenquote wurde entsprechend ebenfalls mehrfach neu berechnet und zuletzt mit $127 \%$ des BIP beziffert. Die griechische Regierung legte im Februar ein Sparprogramm auf, das in der Folge mehrmals verschärft wurde. Die Rating-Agenturen stuften Griechenlands Bonität mehrfach herab, und die wirtschaftliche Lage Griechenlands verschlechterte sich zunehmend: Nach einem starken Rückgang des Bruttoinlandsprodukts um 3,3\% im Jahre 2009 schrumpfte die Wirtschaft 2010 um 3,5\% und 2011 sogar um $6,9 \%$.

Es ist kaum in Abrede zu stellen, dass die wirtschaftspolitischen Fehler in Griechenland gravierend waren; allen voran insbesondere die kaum stattfindende Besteuerung höherer Einkommensgruppen und die zahlreichen Möglichkeiten der Steuerumgehung bzw. -hinterziehung. Davon unabhängig aber war es auf Seiten der anderen Regierungen des Euroraums gleich aus mehreren Gründen ein schwerer wirtschaftpolitischer Fehler, Griechenland nicht schnellstmöglich und unter Auflagen zu unterstützen: Erstens lassen sich gerade tiefgreifende strukturelle Probleme der Wirtschaft und des Steuersystems nicht kurzfristig lösen, sondern benötigen Zeit. Zweitens wurden die Voraussetzungen für eine Lösung der Probleme Griechenlands durch die hohen und steigenden Renditeforderungen der Investoren an den Märkten erschwert. Drittens war es abzusehen, dass der Solidaritätsverzicht die durch die Verluste der internationalen Finanzkrise ohnehin nervösen Marktteilnehmer alarmieren und ihre Bereitschaft schmälern würde, Staatsschuldtitel anderer Euroländer mit hohen Defiziten und/oder hohen Schuldenständen zu halten.

Das Risiko einer Kettenreaktion war auch deshalb besonders groß, weil sich die Industrieländer im ersten Jahr nach der internationalen Finanzkrise befanden. Das

4 ARD, Bericht aus Berlin, 28. Februar 2011. 
Bruttoinlandsprodukt des Euroraums war 2009 trotz starker Zinssenkungen durch die Zentralbank und mehrerer konjunkturstützender fiskalpolitischer Maßnahmen der Regierungen um 4,7 \% gesunken, und die Schuldenstände waren insbesondere durch die Übernahme von Verlusten des Bankensystems in allen Euroländern deutlich gestiegen.

Dennoch zauderten die Regierungschefs des Euroraums im Frühjahr 2010, und das, obwohl die gesamten Staatsschulden Griechenlands lediglich 3,6 \% des Bruttoinlandsprodukts des Euroraums betrugen. Um zehnjährige Staatsanleihen auf dem Kapitalmarkt unterzubringen, musste die griechische Regierung nun $8 \%$ Zinsen bieten (während Deutschlands Anleihen mit $3 \%$ rentierten), und am 23. April sah die griechische Regierung keine andere Möglichkeit, als die EU um finanzielle Hilfe zu bitten. Ende März hatten die Regierungschefs des Euroraums und der Internationale Währungsfonds bereits beschlossen, Griechenland im Notfall eine Summe von $30 \mathrm{Mrd}$. Euro in Form von bilateralen Krediten und IWF-Mitteln zur Verfügung zu stellen; letztlich wurde am 2. Mai 2010 aber ein Hilfspaket mit einem Kreditvolumen in Höhe von 110 Mrd. Euro verabschiedet, dessen Auszahlung an die Erfüllung von strikten Vorgaben geknüpft wurde. So sollte Griechenland sein Budgetdefizit von 13,6 \% des BIP 2009 auf $2 \%$ des BIP im Jahre 2014 zurückführen. Der von Griechenland zu zahlende Zinssatz lag bei 5,5\%, was nicht nur deutlich über den Refinanzierungskosten lag, sondern zudem für ein Land in einer schweren Krise wesentlich zu hoch war. Die Märkte zeigten sich von diesen Maßnahmen nicht beeindruckt. Die Kurse sanken, und die Renditen griechischer Staatsanleihen kletterten Ende April/Anfang Mai an mehreren Tagen über $10 \%$ und erreichten am Freitag, den 7. Mai, mit 12,3 \% ihren vorläufigen Höhepunkt. Auch die Risikoaufschläge auf die Staatsanleihen der anderen Südländer tendierten deutlich nach oben, während die Renditen entsprechender deutscher Papiere nunmehr bei 2,8 \% lagen.

Angesichts der Lage am Markt für Staatsanleihen, die sich insbesondere für die Länder Griechenland, Irland und Portugal zuspitzte, und der Gefahr eines Übergreifens der Krise entschied sich die EZB zum Einschreiten. Am folgenden Montag, dem 10. Mai 2010, intervenierten die Zentralbanken des Eurosystems erstmals am Markt für Staatsanleihen - nur vier Tage, nachdem EZB-Präsident Trichet auf der monatlichen Pressekonferenz erklärt hatte, eine solche Maßnahme wäre während der EZB-Sitzung nicht einmal diskutiert worden. Noch am selben Tag kritisierte der damalige Bundesbankpräsident Axel Weber diesen Beschluss des EZB-Rates in einem Interview mit der Börsen-Zeitung. Obwohl am 10. Mai auch ein EuroSchutzschirm angekündigt wurde, der Garantien und Kreditmittel in Höhe von insgesamt 750 Mrd. Euro von Seiten der Euroländer, des IWF und der EU umfasste, beruhigten sich die Märkte nicht. Im Mai brach dann die Diskussion um eine Zweiteilung des Euroraums aus, einer im Norden und einer im Süden. Die Risikoaufschläge nahmen Woche für Woche weiter zu. Stets hinkten die politischen Entscheidungen der Entwicklung hinterher, stets nahm Deutschland bis zur letzten Minute eine blockierende Haltung ein, und die Beschlüsse, die letztlich unter dem Druck der Kapitalmärkte zustande kamen, waren nicht geeignet, Vertrauen in eine wirtschaftlich stabile Entwicklung im Euroraum zu schaffen. 
Einen erneuten Renditesprung gab es im Oktober 2010, als Kanzlerin Merkel mit der Forderung nach Gläubigerhaftung an die Öffentlichkeit trat. Obwohl ihr Vorstoß den permanenten Krisenmechanismus im Euroraum ab 2013 betraf, wirkte die Ankündigung vor dem Hintergrund der schwierigen wirtschaftlichen und finanziellen Lage insbesondere der südeuropäischen Länder stark verunsichernd auf die Besitzer der Staatspapiere dieser Länder.

EZB-Präsident Trichet und andere warnten vor den Folgen dieser Diskussion und der möglichen Gefahr eines Übergreifens der Vertrauenskrise auf weitere Länder. Bis Mitte November 2010 stiegen die Renditen der Staatsanleihen Griechenlands, Irlands und Portugals auf 11,5 \%, 8,1 \% bzw. 6,8 \%. Noch im selben Monat musste ein Rettungspaket für Irland geschnürt werden, und Irland wurde das erste Land, das unter den im Juni 2010 gegründeten Rettungsschirm EFSF (Europäische Finanzstabilisierungsfazilität) schlüpfte. Bis Ende des Jahres hatten die Zentralbanken des Eurosystems Staatsanleihen von Griechenland, Irland und Portugal im Wert von insgesamt $74 \mathrm{Mrd}$. Euro auf dem Sekundärmarkt gekauft, und alle drei Länder hatten ihren Konsolidierungskurs im Laufe des Jahres nochmals verschärft; die Renditen aber zogen weiter an. Portugal nahm im Mai 2011 als zweites Land Mittel des EFSF und des IWF in Anspruch. Auch die Lage in Griechenland spitzte sich weiter zu. Im Juli 2011 einigten sich die Regierungen der Euroländer auf ein zweites Rettungspaket für Griechenland in Höhe von 109 Mrd. Euro. Gleichzeitig beschlossen sie, die Zinsen der bereits vergebenen Kredite an Griechenland, Irland und Portugal zu senken und im Falle Griechenlands eine private Gläubigerbeteiligung durchzusetzen. Geplant war, dass die Gläubiger sich freiwillig beteiligten und eines von drei Modellen wählten, die unter anderem eine Laufzeitverlängerung und eine Renditeverringerung beinhalteten, mit denen die Gläubiger auf etwa $21 \%$ des Nennwerts ihres Engagements in griechische Staatsanleihen hätten verzichten müssen. Kurz darauf stiegen Investoren auch aus Staatsanleihen Spaniens und Italiens aus. Anfang August 2011 nahmen die Renditen zehnjähriger Staatsanleihen Spaniens und Italiens auf über $6 \%$ Zinsen zu, und die EZB gab bekannt, dass die im Eurosystem zusammengeschlossenen Zentralbanken zur Stabilisierung der Kurse Staatsanleihen dieser beiden Länder aufkaufen würden. Innerhalb von zwei Monaten erhöhte das Eurosystem den Bestand an "aus geldpolitischen Zwecken « erworbenen Staatspapieren um 89 Mrd. Euro auf $163 \mathrm{Mrd}$. Euro. Ohne Zweifel hat sie damit einen noch kräftigeren Anstieg der Renditen und eine noch stärkere Beeinträchtigung des Bankensystems und der Unternehmensfinanzierung verhindert; die Renditen gingen zeitweise sogar um einen Prozentpunkt zurück. Im September 2011 verabschiedete Spaniens Parlament einen Verfassungszusatz, der die Regierung auf eine strikte Defizitbegrenzung verpflichtete, und Italien beschloss ein dreijähriges öffentliches Sparprogramm. Für den Euroraum insgesamt zeichnete sich derweil eine Rezession $\mathrm{ab}$, und die Rating-Agenturen führten nun nicht mehr überhöhte Schulden, sondern die schlechten Konjunkturaussichten als Hauptgrund für Herunterstufungen der Länder-Ratings im Euroraum an. Deutschland als »sicherer Hafen " musste im Herbst 2011 für einen Wertpapierkredit des Staates mit zehnjähriger Laufzeit nur noch knapp 2 \% Zinsen zahlen. Im restlichen Euroraum spitzte sich die Lage dagegen weiter zu. Griechenland wurde die Auszahlung einer weiteren Tranche des ers- 
ten Rettungspakts zunächst verweigert, die Aktienkurse fielen, für zahlreiche Euroländer stiegen die zu zahlenden Zinsen für Staatsanleihen weiter, und es häuften sich dramatische Appelle aus der Politik. EU-Kommissionspräsident Barroso erklärte, die Union stehe vor ihrer bisher größten Herausforderung, IWF-Chefin Lagarde mahnte die Euroländer, »jetzt zu handeln und zusammen zu handeln «, Kanzlerin Merkel und EZB-Präsident Trichet sprachen von der »schwersten Krise seit dem 2. Weltkrieg «, und US-Finanzminister Geithner forderte die Euroländer auf, »eine Brandmauer " zu errichten, um ein weiteres Ausbreiten der Krise zu verhindern.

Aber wieder blieb der große Wurf der Politik aus, und wieder war es die EZB, die als Retterin in der Not in Aktion trat. Anfang Oktober 2011 kündigte sie ein einjähriges Refinanzierungsgeschäft mit den Banken an. Die Regierungen gaben nun zwar grünes Licht für die nächste Tranche an Griechenland, einigten sich Ende Oktober auf eine Erhöhung der Mittel des Rettungsfonds und kündigten Anfang Dezember einen Fiskalpakt an; ein sichtbarer stabilisierender Effekt ging hiervon allerdings nicht aus. Das mag auch daran liegen, dass die im Juli beschlossene private Gläubigerbeteiligung im Falle Griechenlands im Oktober kurzerhand von knapp $25 \%$ auf $50 \%$ erhöht wurde (letztlich waren es Anfang März 2012 dann 53,5 \% ohne die Möglichkeit, eine der im Juli 2011 vorgestellten Optionen zu wählen). Ende November 2011 rentierten zehnjährige Staatspapiere Italiens bei über $7 \%$, jene Griechenlands und Portugals bei 29 \% bzw. 13 \%. Im Euroraum drohte zudem eine Kreditklemme.

Vor diesem Hintergrund ergriff die EZB im Dezember 2011 abermals weitreichende Maßnahmen. Sie kündigte insbesondere zwei dreijährige Refinanzierungsgeschäfte für die Banken an und verringerte die Anforderungen an die zu hinterlegenden Sicherheiten. Auch halbierte sie die Mindestreservepflicht der Banken auf $1 \%$ und senkte den Leitzins, der bereits im November um einen Viertelprozentpunkt gesenkt worden war, auf $1 \%$ und damit auf das Niveau, das erst im April 2011 mit einer Zinserhöhung verlassen worden war.

\section{Schiefe Verteilung der Refinanzierungsgeschäfte und hohe TARGET2-Salden}

Die konventionellen und unkonventionellen geldpolitischen Maßnahmen, die die EZB im Zuge der Krise ergriffen hat, haben verhindert, dass es zu einer noch stärkeren Zuspitzung der Krise kam, und damit wohl auch, dass der Euroraum auseinanderbrach. Sie haben aber auch dazu geführt, dass die EZB und die nationalen Zentralbanken des Eurosystems ein hohes Volumen an risikobehafteten Aktiva in ihren Bilanzen stehen haben. Dies sind zum einen die Staatsanleihen, die das Eurosystem seit Mai 2010 gekauft hat, um mäßigend auf die langfristigen Zinsen zu wirken. Ende Juni beliefen sich diese auf $211 \mathrm{Mrd}$. Euro und hatten einen Anteil an der konsolidierten Bilanz des Eurosystems von $7 \%$. Hinzu kommen Pfandbriefe, die aus ähnlichen Gründen im Wert von $70 \mathrm{Mrd}$. Euro erworben wurden. Ein weit höheres Gewicht aber haben die Refinanzierungsgeschäfte mit den Banken. Diese nahmen bereits während der Finanzkrise 2007/2009 dramatisch zu. Damals waren nahezu alle Banken betroffen. Da die Banken das Vertrauen in die Zahlungsfähig- 
keit anderer Banken verloren hatten, liehen sie sich gegenseitig - anders als sonst üblich - kaum Geld am Geldmarkt. Folglich mussten sich die Banken stärker bei der Zentralbank refinanzieren. Mit der Bewältigung der internationalen Finanzkrise kehrte das Vertrauen zurück, und der Refinanzierungsbedarf verringerte sich. Im Zuge der Staatsanleihenkrise im Euroraum erhöhte sich der Refinanzierungsbedarf beim Eurosystem dann wieder und erhielt mit jeder Verschärfung der Vertrauenskrise einen weiteren Schub. Im Rahmen der zwei dreijährigen niedrig verzinsten Refinanzierungsgeschäfte im Dezember 2011 und im März 2012 liehen sich die Banken insgesamt 1.019 Mrd. Euro vom Eurosystem. Da teilweise kürzer laufende Refinanzierungskredite abgelöst wurden, erhöhte sich das Refinanzierungsvolumen um nahezu $500 \mathrm{Mrd}$. Euro und befindet sich mit einem Volumen von rund 1.200 Mrd. Euro (Juni 2012) auf einem Allzeit-Hoch (Abbildung 2).

\section{Abbildung 2: Konsolidierte Bilanz des Eurosystems}

Januar 2007 bis Juni 2012,1 in Mrd. Euro

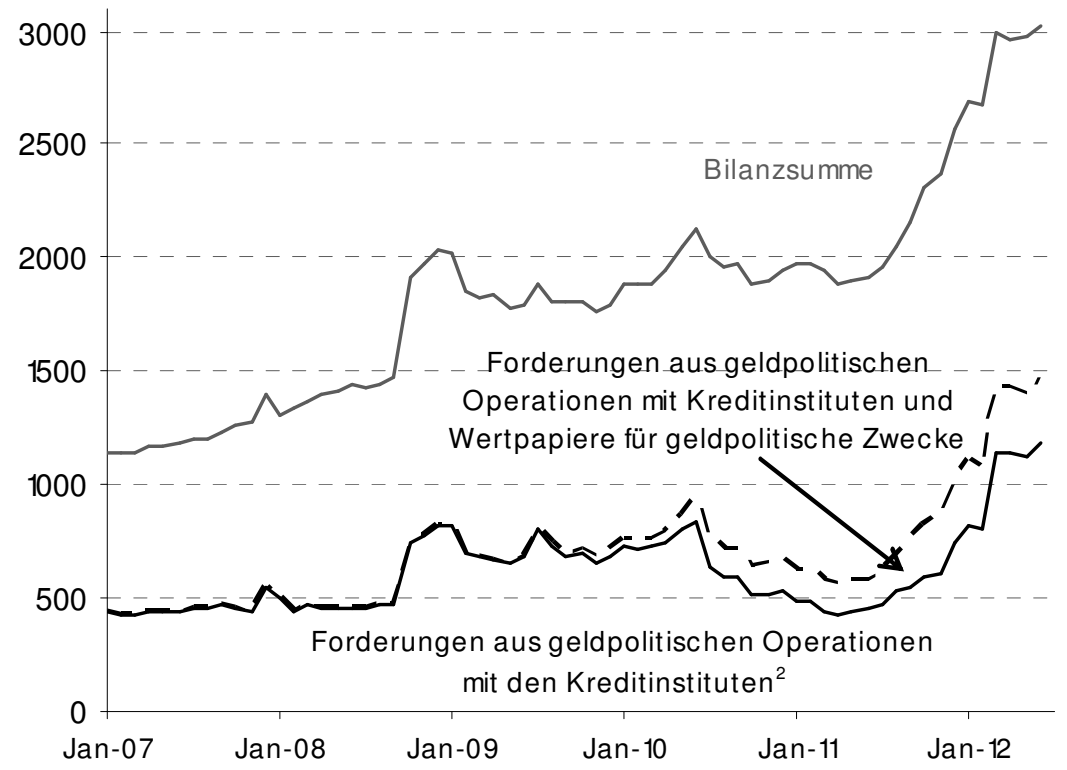

\footnotetext{
${ }^{1}$ Monatsdurchschnitte der ausgewiesenen Wochenendwerte.

${ }^{2}$ Hauptrefinanzierungsgeschäfte, längerfristige Refinanzierungsgeschäfte, Feinsteuerungsoperationen in Form von befristeten Transaktionen, strukturelle Operationen in Form von befristeten Transaktionen, Spitzenrefinanzierungsfazilität und Forderungen aus Margenausgleich.
}

Quellen: Europäische Zentralbank; eigene Berechnungen.

Anders als während der internationalen Finanzkrise sind nicht alle Banken gleichermaßen abhängig von den Refinanzierungsmitteln der Zentralbank. Es sind in erster Linie die Banken in den Krisenländern, die sich infolge schrumpfender Ein- 
lagen und eines eingeschränkten Zugangs zum Geldmarkt verstärkt beim Eurosystem refinanzieren. So stiegen die den griechischen Banken zugeteilten Refinanzierungsmittel der Zentralbank zwischen Januar 2008 und Januar 2012 von 4 Mrd. auf $74 \mathrm{Mrd}$., die Irlands von $31 \mathrm{Mrd}$. auf $93 \mathrm{Mrd}$. und jene spanischer und italienischer Banken um 120 Mrd. bzw. 188 Mrd. Euro. Insgesamt beanspruchten Griechenland, Irland, Italien, Spanien und Portugal im Januar 201271 \% der durch Offenmarktgeschäfte zugeteilten Zentralbankliquidität, verglichen mit $17 \%$ im Januar 2008. Demgegenüber haben die Banken anderer Euroländer, insbesondere Deutschlands, ihre Verschuldung beim Eurosystem verringert, weil sie einen Mittelzufluss aus eben diesen Ländern verzeichneten. Hatten deutsche Banken im Januar 2008 noch einen Refinanzierungsbedarf von 173 Mrd. Euro, so betrug er im Mai 2012 nur noch 80 Mrd.; der Anteil an den gesamten Refinanzierungsmitteln des Eurosystems sank entsprechend von $40 \%$ auf $10 \%$.

Der steile Anstieg der Nachfrage nach Refinanzierungsmitteln des Eurosystems ist primär die Folge mangelnder alternativer Refinanzierungsmöglichkeiten der Banken der Krisenländer. Nicht nur sind Interbanken-Kredite Mangelware, sondern Inländer ziehen zudem ihre Einlagen ab. Dieser Abfluss von Kapital spiegelt sich in den Forderungen und Verbindlichkeiten zwischen den nationalen Zentralbanken des Eurosystems wider, die in dem Verrechnungssystem TARGET2 verbucht werden. Wenn private Gläubiger beispielsweise Mittel von einer italienischen Bank abziehen und auf einer deutschen Bank einzahlen, handelt es sich um eine grenzüberschreitende Transaktion, die die Zentralbanken Italiens und Deutschlands berührt. Sofern diese Positionen bis zum Ende jedes Tages nicht durch gegenläufige Transaktionen ausgeglichen werden, werden diese in Forderungen und Verbindlichkeiten gegenüber der EZB umgewandelt. Vor der internationalen Finanzkrise waren diese TARGET- bzw. TARGET2-Salden vernachlässigbar. Im Mai 2012 - dem aktuellsten zugänglichen Datenstand - erreichten sie Rekordwerte: Die TARGET2-Forderungen der Bundesbank beliefen sich auf 699 Mrd. Euro, Italien und Spanien hatten TARGET2-Verbindlichkeiten in Höhe von 279 Mrd. Euro bzw. 303 Mrd. Euro (April 2012) (Abbildung 3).

Vor dem Hintergrund der aktuellen Diskussion in Deutschland ist es wichtig zu betonen, dass es keinen kausalen Zusammenhang zwischen der Leistungsbilanzposition eines Landes und dem Vorzeichen seines TARGET2-Saldos gibt. ${ }^{5}$ Italien beispielsweise verzeichnete seit 2002 Leistungsbilanzdefizite, zugleich jedoch einen positiven TARGET2-Saldo bis Juli 2011; erst zu diesem Zeitpunkt wurde Italien von der Ansteckungswelle im Euroraum erfasst. Seither haben die TARGET2-Verbindlichkeiten der Banca d'Italia in gigantischen Schritten zugenommen. Ein weiteres Beispiel für den nichtexistenten Zusammenhang zwischen Leistungsbilanzsaldo und TARGET2-Saldo liefert Irland. Irland hat es bereits 2010 geschafft, seine Leistungsbilanz in den positiven Bereich zu bringen. Dennoch verbleiben die TAR-

5 Einige Volkswirte in Deutschland argumentieren, dass es einen derartigen Zusammenhang gibt; siehe Sinn, Wollmershäuser 2011. Olaf Storbeck und Buiter et al. gehörten zu den Ersten, die auf die logischen Fehler dieser Argumentation hinwiesen; Buiter et al. 2011 a, 2011 b; Storbeck 2011; siehe auch Tober 2011. 


\section{Abbildung 3: TARGET2-Salden ausgewählter nationaler Zentralbanken des Eurosystems 1 \\ Januar 2007 bis Mai 2012, Monatsendwerte, in Mrd. Euro}

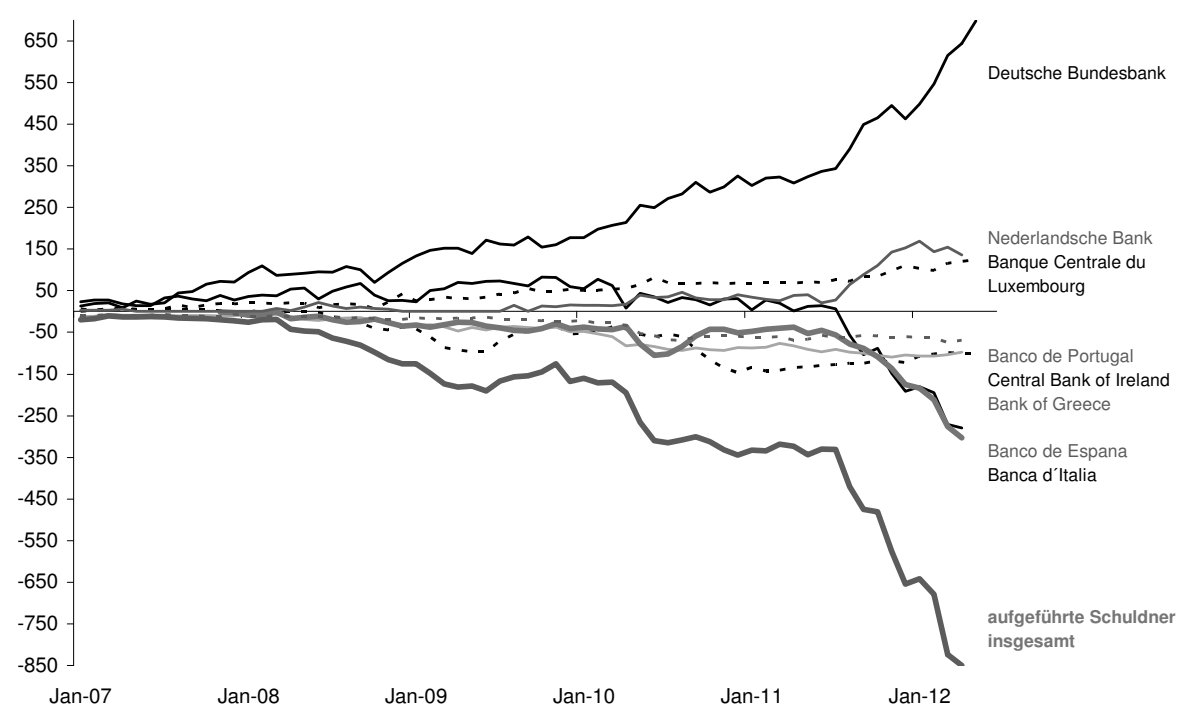

${ }^{1}$ Die Datenreihen entstammen den Bilanzausweisen der nationalen Zentralbanken wie folgt: Deutschland - TARGET2 (netto) (Zeitreihe EU8148 b); Griechenland - TARGET2- bzw. TARGET-Verbindlichkeiten; Finnland - Forderungen in Zusammenhang mit TARGET (netto); Irland - »Andere Verbindlichkeiten innerhalb des Eurosystems " approximiert durch »Andere Verbindlichkeiten " minus knapp $20 \mathrm{Mrd}$. Euro als Proxy für sonstige Verbindlichkeiten und Verbindlichkeiten aus der Zuteilung von Banknoten; ItalienAndere Forderungen/Verbindlichkeiten innerhalb des Eurosystems (netto); Luxemburg - Forderungen der Zentralbank innerhalb des ESZB; Niederlande - Intra-ESZB-Forderungen; Portugal - Täglich fällige Verbindlichkeiten gegenüber MFIs in anderen Euroraum-Ländern; Spanien -Einlagen MFIs anderer EuroraumLänder (Reihe 7.94).

Quellen: Bilanzausweise der nationalen Zentralbanken.

GET2-Salden mit rund 100 Mrd. Euro (Mai 2012) auf sehr hohem Niveau und übersteigen bei weitem die kumulierten Leistungsbilanzdefizite der Vergangenheit. TARGET2-Salden haben dieselbe Wurzel wie die gestiegene Abhängigkeit von Zentralbankliquidität - ein Mangel an Vertrauen in die Solvenz des Bankensystems der Krisenländer. Es ist daher auch nicht erstaunlich, dass die Refinanzierungsgeschäfte des Eurosystems und die TARGET2-Salden ähnliche Größenordnungen aufweisen. ${ }^{6}$ Da TARGET2-Salden Forderungen und Verbindlichkeiten gegenüber der EZB darstellen, werden Verluste gegebenenfalls entsprechend des Kapitalanteils an der EZB auf die nationalen Zentralbanken verteilt. Sollte eines dieser Länder den Euroraum verlassen, so ist es äußerst unwahrscheinlich, dass es in der Lage und gewillt sein wird, seine TARGET2-Verbindlichkeiten zu begleichen, zumal der Euro gegenüber der neuen Währung des Landes deutlich aufgewertet sein dürfte.

6 Tober 2011. 


\section{Der Fiskalpakt: notwendiges Übel oder Irrweg?}

Die Risiken, die die EZB im Zuge ihrer Stabilisierungspolitik eingegangen ist, sind groß. Sie einzugehen war jedoch erforderlich, um den Euroraum zusammenzuhalten. Der EZB fehlt aber letztlich die demokratische Legitimation, derart hohe Risiken einzugehen, auch wenn ihre Analyse richtigerweise nahelegt, dass ein Nichthandeln mit noch höheren möglichen Kosten verbunden wäre. Zudem kann die EZB - anders als die Regierungen - keine Bedingungen an die Unterstützung knüpfen. ${ }^{7}$ Dies dürften auch die Gründe dafür sein, dass EZB-Präsident Jean-Claude Trichet und sein Nachfolger Mario Draghi fortwährend die Regierungen zu stärkerem Handeln aufgefordert haben. Im Frühjahr und Frühsommer 2011 drohte Trichet gar damit, die unterstützenden Maßnahmen einzustellen, wenn die Politik ihrer Verantwortung nicht nachkäme - eine Kritik, die sein Nachfolger Mitte November, bezogen auf die volle Funktionsfähigkeit des Rettungsschirms, ebenfalls vehement äußerte: »Where is the implementation of these long-standing decisions? « .8

Aus Sicht der EZB bestand die Verantwortung der Regierenden darin, die Schlagkraft des Rettungsschirms zu erhöhen und verpflichtende Regeln zum Schuldenabbau und zur Defizitbegrenzung zu verabschieden. Da der Rettungsschirm eine Haftung für die Schulden einzelner Länder durch die anderen Länder beinhaltet, ist es folgerichtig, dass er an Bedingungen geknüpft ist und auch Regelungen gefunden werden, die die Gefahr einer künftigen Überschuldung eines Eurolands möglichst gering halten. Was die Regierungen aber mit dem erhöhten Volumen des Rettungsschirms und der Verabschiedung des Fiskalpakts Anfang März 2012 auf den Weg gebracht haben, ist weder geeignet, die gegenwärtige Krise zu beenden, noch dazu, dem Euroraum den Weg in eine stabile wirtschaftliche Entwicklung zu bahnen.

\section{Rettungsschirm zu klein}

Der Rettungsschirm ist angesichts der Höhe der ausstehenden Staatsschulden der von der Krise derzeit am stärksten betroffenen Länder zu gering. Die Staatsverschuldung Griechenlands, Irlands, Italiens, Portugals, Spaniens und Zyperns beträgt insgesamt rund $3.500 \mathrm{Mrd}$. Euro. Kämen weitere Länder hinzu, wäre der Bedarf entsprechend höher. Zudem soll der Europäische Stabilitäts-Mechanismus (ESM) gegebenenfalls nicht nur die Staaten selbst, sondern auch die Banken mit Krediten unterstützen. Die veranschlagten potentiellen Rettungsmittel belaufen sich aber nur auf $800 \mathrm{Mrd}$. Euro, von denen rund die Hälfte bereits als Hilfen an Griechenland, Irland und Portugal und das spanische Bankensystem vergeben bzw. vorgemerkt sind. Die 800 Mrd. Euro setzen sich zusammen aus 500 Mrd. Euro, die dem permanenten Rettungsschirm ESM zur Verfügung stehen werden. Hinzu kommen die bereits vergebenen Kredite des provisorischen Rettungsschirms EFSF in Höhe von rund 200 Mrd. Euro sowie rund 100 Mrd. Euro des ersten Griechenlands-Pakets,

7 Die EZB kann lediglich, wie der Brief von Jean-Claude Trichet und Mario Draghi an die italienische Regierung im September 2011 zeigte, vereinzelt Druck ausüben; siehe Financial Times Deutschland 2011. EZB nimmt Italien in die Zange. 6. September 2011, S. 1.

8 Draghi 2011.

Leviathan, 40. Jg., 3/2012 
die noch vor Inkrafttreten des EFSF aus diversen EU-Töpfen sowie auf bilateraler Basis vergeben wurden.

Vielfach werden bei der Beurteilung der Schlagkraft des Rettungsschirms die verfügbaren Kreditmittel mit der Neuverschuldung und dem Refinanzierungsbedarf der Krisenländer in den kommenden Jahren verglichen. Dieser Vergleich ist insofern wichtig, als er anzeigt, ob der Fonds eine unmittelbare Zahlungsunfähigkeit verhindern kann. Entscheidend für die Stabilisierung des Euroraums ist aber der Vergleich mit der gesamten Staatsverschuldung dieser Länder, da sich ein Kursverfall bzw. ein Renditeanstieg dieser Wertpapiere auch auf die finanzielle Lage der Banken und der Unternehmen auswirkt. Solange die Gefahr eines Schuldenschnitts nicht gebannt ist, besteht auch die Gefahr einer Belastung des heimischen Bankensystems und der heimischen Unternehmen. Und das Risiko eines Schuldenschnitts beinhaltet zugleich das Risiko eines Austritts aus dem Euroraum und damit die Einführung einer neuen Währung. Auch dies macht die Anleihen von Banken und Unternehmen der Krisenländer riskant und spiegelt sich in erhöhten Risikoaufschlägen wider. Eben aus diesem Grund muss ein Rettungsschirm ein unbegrenztes Einsatzvolumen haben. Das wiederum kann (ohne die sofortige Einführung von Eurobonds einschließlich der Konvertierung aller bisherigen Schulden in Eurobonds) nur von der EZB geleistet werden. Erklärte die EZB, dass sie die Kurse der Staatsanleihen verteidigen und nur noch minimale Renditedifferentiale akzeptieren wird, so dürfte es zu einer sofortigen Stabilisierung der Kurse kommen. Günstigenfalls müsste sie gar keine Mittel einsetzen. Sinnvollerweise würde das Eurosystem vor der Ankündigung in großem Stil Staatsanleihen der Krisenländer kaufen und so im Zuge des folgenden Kursanstiegs Gewinne realisieren, die dann an die Kapitaleigner der EZB, die Regierungen der Euroländer, ausgeschüttet würden und damit dem Konsolidierungsprozess zugute kämen. Wie bereits weiter oben ausgeführt, hat die EZB ohne politische Rückendeckung allerdings nicht das Mandat, eine solch weitreichende Ankündigung zu machen. Wie weiter unten näher ausgeführt wird, bedarf es einer politischen Rückendeckung, die die Entschlossenheit der Regierungen bekundet, den Euroraum in seiner jetzigen Zusammensetzung zu erhalten und den Mitgliedsländern zu helfen, ihre Zahlungsfähigkeit aufrechtzuerhalten, und mit der zudem nicht gegen die Urteile des Bundesverfassungsgerichts verstoßen wird.

\section{Fiskalpakt zu eng}

Während der Rettungsschirm vom Volumen her weiterhin inadäquat ist, ist der Fiskalpakt weder vom Inhalt her wirklich neu noch geeignet, Stabilität zu fördern. Die wesentlichen Aussagen des Fiskalpakts finden sich bereits in dem 1999 in Kraft getretenen Stabilitäts- und Wachstumspakt, demzufolge das Defizit- und das Schuldenkriterium des Maastricht-Vertrags nicht nur zum Zeitpunkt des Beitritts, sondern auch in der Folge eingehalten werden müssen. Die entsprechenden MaastrichtKriterien lauteten $3 \%$ als maximales Defizit der öffentlichen Haushalte und $60 \%$ als maximaler Schuldenstand des Staates (jeweils in \% des Bruttoinlandsprodukts). Der Stabilitäts- und Wachstumspakt stellte insofern eine Verschärfung dar, als die Staaten angehalten waren, in der mittleren Frist einen annähernd ausgeglichenen 
oder überschüssigen Haushalt anzustreben. Mit der Reform des Stabilitätspakts im Jahre 2005 wurde dieser einerseits etwas flexibler, zugleich wurden aber die Durchsetzungsmöglichkeiten ausgebaut. Hinzu kamen die sogenannten »Six-Pack «-Vorschriften, die im Dezember 2011 in Kraft getreten sind. Hierdurch wurde auch das Schuldenstandskriterium so verschärft, dass nun auch Sanktionen gegen ein Land verhängt werden können, das zwar nicht gegen das Defizitkriterium verstößt, aber seine über $60 \%$ liegende Schuldenstandsquote nicht schnell genug reduziert, und zwar jährlich um ein Zwanzigstel des Differenzbetrags zu $60 \%$. Das Neue am Fiskalpakt, auf den sich 25 der 27 Regierungschefs Anfang März 2012 verständigten, liegt darin, dass er in nationales Recht umgesetzt werden soll - möglichst mit Verfassungsrang - und dass die umgekehrte qualifizierte Mehrheitsregel (ein Beschluss gilt als angenommen, es sei denn, eine qualifizierte Mehrheit votiert dagegen) eine weitere Anwendung findet.

Eine nachhaltige Fiskalpolitik ist eine wesentliche Voraussetzung für eine stabile wirtschaftliche Entwicklung. Dies ist in einer Währungsunion umso wichtiger, als Fehlentwicklungen in einem Mitgliedsstaat stärker auf die anderen Länder ausstrahlen und letztlich eine wie auch immer geartete Rettungsaktion (Bailout) im schlimmsten Fall unvermeidbar wäre. Der Fiskalpakt hätte die aktuellen Probleme im Euroraum aber nicht verhindert und wird auch nicht zur Überwindung der aktuellen Krise beitragen können. Die Hoffnung der Regierungschefs und auch der EZB ist zwar, dass der Fiskalpakt entscheidend dazu beitragen wird, das Vertrauen in die Nachhaltigkeit der Staatsfinanzen im Euroraum zu stärken und damit auch das Vertrauen der Investoren in die Staatsanleihen der Krisenländer. In der kurzen bis mittleren Frist wird die Umsetzung des Fiskalpakts aber dämpfend auf die wirtschaftliche Aktivität wirken und könnte durch die resultierende Schwächung des Bankensystems und die verringerten Steuereinahmen zu einem erneuten Vertrauenseinbruch führen. Da außer Luxemburg und Finnland derzeit alle Länder des Euroraums ein strukturelles - also um konjunkturelle Einflüsse bereinigtes - Defizit aufweisen, das größer ist als die vom Fiskalpakt vorgeschriebenen $0,5 \%$ des BIP, bzw. einen zu hohen Schuldenstand haben, müssen diese Staaten alle gleichzeitig Ausgaben kürzen bzw. Einnahmen erhöhen. Sie können damit in der aktuell schwierigen wirtschaftlichen Lage des Euroraums nicht einmal die automatischen Stabilisatoren voll wirken lassen, also die stabilisierende fiskalische Wirkung, die durch konjunkturbedingt höhere Ausgaben wie beispielsweise Arbeitslosengeldzahlungen bei zugleich konjunkturbedingt geringeren Steuereinnahmen entsteht. Man könnte zwar argumentieren, die Geldpolitik sei gegenwärtig ungewöhnlich expansiv und kompensiere daher die kontraktiven Impulse der Fiskalpolitik; in weiten Teilen des Euroraums stimuliert die lockere Geldpolitik aber eben nicht Investitionen und Konsum, sondern verhindert lediglich wirtschaftlichen Zusammenbruch angesichts einer zunehmenden Kapitalflucht und angesichts von Banken, deren Bilanzen durch die massiven Kursverluste der Staatspapiere und die schlechte Konjunktur sowie teilweise deutliche Verluste bei Immobilienkrediten in Schieflage geraten sind. Insgesamt waren die Buchkredite der Banken an private Haushalte und Unternehmen im Euroraum zuletzt mit einer Rate von -0,1\% leicht rückläufig (Mai 2012), in Griechenland, Irland, Portugal und Spanien sogar sehr deutlich. 
Zusätzlich zu dieser negativen Wirkung des Fiskalpakts auf die wirtschaftliche Aktivität gibt es mindestens drei weitere Aspekte, die die Frage aufwerfen, ob der Fiskalpakt geeignet ist, Vertrauen zu stiften. Erstens stellt sich die Frage, ob die ehemals in der deutschen Verfassung enthaltene Regelung nicht sinnvoller ist, nach der das Defizit die Höhe zukunftsgerichteter öffentlicher Investitionen nicht überschreiten solle. Warum sollten heutige Steuerzahler in vollem Umfang für Investitionen zahlen, die künftigen Generationen zugute kommen? Zweitens impliziert die Defizitregel des Fiskalpakts eine langfristige Schuldenstandsquote (Schuldenstand in \% des Bruttoinlandsprodukts), die deutlich unter der 60-Prozentmarke des Maastricht-Vertrags liegt. Unterstellt man ein nominales Wachstum von 3,5 \% (1,6 \% reales Wachstum zuzüglich 1,9\% Inflation), so würde der Schuldenstand bei einem erlaubten jährlichen strukturellen Defizit von $0,5 \%$ auf $14 \%$ des Bruttoinlandsprodukts sinken. ${ }^{9}$ Allerdings sieht der Fiskalpakt vor, dass Länder mit einer Schuldenstandsquote von unter $60 \%$ ein strukturelles Defizit von $1 \%$ des Bruttoinlandsprodukts haben können. Dieser Vorgabe zufolge würde die Schuldenstandsquote des Staates auf knapp $30 \%$ sinken. In der Literatur gibt es keine Hinweise darauf, dass eine Schuldenstandsquote von $30 \%$ optimal ist - es gibt daher auch keinen begründeten Anlass, dieses Ziel anzustreben. Im Falle Deutschlands, das mit einer Schuldenstandsquote von 83 \% startet, wäre bei sofortiger Einhaltung des Fiskalpakts eine Schuldenstandsquote von $60 \%$ nach 13 Jahren erreicht, eine Schuldenstandsquote nahe $30 \%$ nach etwa 70 Jahren. Wäre es demgegenüber das fiskalpolitische Ziel, eine Schuldenstandsquote von $60 \%$ zu verankern, so müsste - bei einem nominalen Wachstum von 3,5\% - das jährliche strukturelle Defizit $2 \%$ betragen! Entsprechend konvergiert die Schuldenstandsquote bei einem strukturellen Defizit von 0,5\% nur dann gegen $60 \%$, wenn das reale Wachstum zuzüglich Inflationsrate knapp 0,9 \% beträgt. Aber auch ein Schuldenstand von $60 \%$ hat keine besonderen wirtschaftlichen Eigenschaften. Mit einem strukturellen Defizit von 2,7\% würde man bei einem nominalen Wachstum von 3,5\% eine Schuldenstandsquote von $80 \%$ stabilisieren.

Problematisch an einem hohen Schuldenstand ist in erster Linie, dass er zwangsläufig mit einer hohen Zinsbelastung einhergeht, sodass ein entsprechend hoher Teil der Steuereinnahmen nicht für andere Zwecke zur Verfügung steht. Ferner ist die Gefahr, durch unerwartete schwerwiegende Ereignisse in eine Situation zu geraten, in der die Nachhaltigkeit der öffentlichen Finanzen nicht mehr zu gewährleisten ist, größer, je höher der Ausgangsschuldenstand ist. Das alles kann aber kein Grund sein, eine Schuldenstandsquote von $30 \%$ völkerrechtlich zu verankern. Auch eine Zweidrittelmehrheit des Bundestags kann dann nicht mehr beschließen, Deutschland von der Regel zu entbinden, einen ausgeglichenen Haushalt bzw. Budgetüberschüsse in der mittleren Frist zu erzielen. Eine weniger willkürliche und ökonomisch begründete Regel ist die sogenannte »Goldene Regel «, die früher Bestandteil des

9 Für Deutschland ist die in der Verfassung verankerte Schuldenbremse, die ab 2016 ein strukturelles Defizit des Bundes von nur 0,35 \% erlaubt, noch strikter. Unterstellt man wie oben ein nominelles Wachstum des Bruttoinlandsprodukts von 3,5\%, impliziert dies eine Konvergenz des Schuldenstands auf $10 \%$ des BIP. 
deutschen Grundgesetzes war und derzufolge das strukturelle Defizit die öffentlichen Investitionen nicht überschreiten darf.

Schwerwiegender noch ist, drittens, dass das strukturelle Defizit - Kernstück des Fiskalpakts - nicht eindeutig zu beziffern ist. Auch wenn man es nach derselben Methode und für dasselbe Jahr berechnet, fällt das strukturelle Defizit unterschiedlich aus, je nachdem, in welchem Jahr es berechnet wird. Für Deutschland beispielsweise wies der Internationale Währungsfonds im Oktober 2001 für das Jahr 1999 ein strukturelles Defizit in Höhe von 0,7 \% aus. Im April 2005 bezifferte der Internationale Währungsfonds das strukturelle Defizit für dasselbe Jahr, also 1999, mit 1,9\%, im April 2008 mit 1,0 \% und im April 2012 mit 1,4 \%. Revisionen des vom Statistischen Bundesamt berechneten Bruttoinlandsprodukts spielen hier eine gewisse Rolle, entscheidend ist aber die Berechnung des Produktionspotentials. ${ }^{10}$ Für die Berechnung des Produktionspotentials benötigt man eine Vielzahl von Größen, die nicht beobachtbar und folglich nur schwer zu quantifizieren sind, wie die Anzahl der potentiellen Erwerbstätigen, deren potentielle Arbeitszeit, die potentielle Produktivität und einiges mehr. Auch komplizierte Verfahren, die beispielsweise Produktionsfunktionen verwenden, kommen letztlich ohne vereinfachende Methoden nicht aus. Letztere bestehen im Wesentlichen in statistischen Glättungsfiltern, für die der aktuelle Rand, also die Gegenwart, und die daraus abgeleiteten Prognosen für die nahe Zukunft und die nahe Vergangenheit eine große Rolle spielen.

Ist das aktuelle Wachstum hoch, so ergeben Schätzungen auch ein relativ hohes Wachstum des Produktionspotentials, und entsprechend gering wird das strukturelle Defizit ausgewiesen - ein Problem, auf das jüngst auch die EZB hingewiesen hat. ${ }^{11}$ Blickt man, wie in Deutschland im Jahre 2005, auf eine längere Schwächephase zurück, ist auch das errechnete Produktionspotential gering, und folglich wird das strukturelle Defizit des Staates höher ausgewiesen als in wirtschaftlich kräftigeren Zeiten. Diese Problematik hat zur Folge, dass der Fiskalpakt ähnlich wie die mit Verfassungsrang ausgestaltete Schuldenbremse Deutschlands tendenziell prozyklisch wirkt: In wirtschaftlichen Hochphasen wird das Produktionspotential hoch ausgewiesen und das strukturelle Defizit folglich gering, so dass der Staat problemlos etwas mehr ausgeben kann - und damit konjunkturverstärkend wirkt. In Schwächephasen wird das Produktionspotential jedoch nach unten angepasst, sodass der Konsolidierungsbedarf des Staates steigt und er entsprechend in die Schwächephase hinein mehr sparen muss und diese dadurch verschärft.

Die kurz- bis mittelfristig wirtschaftlich dämpfende Wirkung des Fiskalpakts, die willkürliche Festlegung auf einen langfristigen Schuldenstand von rund 30\% des Bruttoinlandsprodukts und seine prozyklische Wirkung sind bedenklich genug. Darüber hinaus adressiert der Fiskalpakt aber nicht einmal die Ursache für die Ungleichgewichte im Euroraum und damit eine wichtige Ursache der aktuellen Krise. Vom Fiskalpakt gehen somit nicht nur destabiliserende Effekte in der aktuellen Situation aus, er kann zudem ungleichgewichtige Entwicklungen, wie sie den Euroraum seit seiner Gründung zeichneten, nicht verhindern.

10 Horn, Tober 2007.

11 Europäische Zentralbank 2012, S. 115 ff.

Leviathan, 40. Jg., 3/2012 
Hätte der Fiskalpakt bereits 1999 bestanden, so hätten die heutigen Krisenländer Spanien und Irland bis 2007 keine Probleme gehabt, ihn einzuhalten. Irlands Schuldenstand, der $199948 \%$ des BIP betrug, sank bis 2007 auf $25 \%$ des BIP. Irlands öffentliche Haushalte hätten den Vorgaben des Fiskalpakts zufolge somit ein strukturelles Defizit in Höhe von $1 \%$ aufweisen dürfen. Nach Angaben des World Economic Outlook vom April 2008 verzeichneten die öffentlichen Haushalte aber mit Ausnahme des Jahres 2002 stets sogar einen strukturellen Überschuss. ${ }^{12}$ Spanien reduzierte seine Schuldenstandsquote von $62 \%$ im Jahre 1999 auf $36 \%$ im Jahre 2007. Auch Spanien wies aus Sicht des Jahres 2008 überwiegend strukturelle Budgetüberschüsse auf und hätte lediglich in den Jahren 1999 und 2001 mit strukturellen Defiziten in Höhe von 1,3 \% bzw. 1,2 \% leicht gegen den Fiskalpakt verstoßen. In beiden Ländern war es primär die private Verschuldung, die massiv zunahm und die ungleichgewichtige Entwicklung erzeugte, die der gegenwärtigen Krise zugrunde liegt. Die Regelungen des Fiskalpakts hätten das Problem weder frühzeitig angezeigt noch verhindert.

\section{Das Dauerproblem der EZB: ein gespaltener Euroraum}

Der vehementeste Kritiker der krisenbedingt hohen TARGET2-Salden der Zentralbanken des Eurosystems, Hans-Werner Sinn, war einer jener Ökonomen, die in den ersten Jahren der Währungsunion argumentierten, die jahrelang über dem Inflationsziel der EZB liegende Inflationsrate Spaniens sei kein makroökonomisches Problem, da Spanien ein Land mit aufholender Entwicklung und folglich stark steigenden Dienstleistungspreisen sei. ${ }^{13}$ (Ein Land mit aufholender Entwicklung hat ein überproportionales Produktivitätswachstum in den industriellen Sektoren und ein entsprechend steigendes Lohnniveau. Sofern die Löhne auch im Dienstleistungssektor stärker anziehen, obwohl hier meist keine hohen Produktivitätszuwächse zu verzeichnen sind, steigt die Inflationsrate, ohne dabei ein Ungleichgewichtsphänomen zu sein. Dieser Zusammenhang wird Balassa-Samuelson-Effekt genannt.)

Die überdurchschnittlichen Inflationsraten in Spanien und auch in Portugal, Griechenland und Irland waren aber keine unbedenkliche Nebenerscheinung eines Aufholprozesses, sondern Ausdruck einer konjunkturellen Überhitzung: Die Produktivität in der Industrie nahm in diesen Ländern - und insbesondere in Spanien - nicht überdurchschnittlich stark zu. ${ }^{14}$ Bei einer Orientierung am Inflationsziel der EZB von knapp 2 \% hätten die nominalen Lohnstückkosten in allen Ländern des Euroraums zwischen 1999 und 2007 um rund 18 \% steigen müssen - dies war lediglich in Frankreich annähernd der Fall. Demgegenüber stiegen sie in Griechenland, Portugal und Spanien um $27 \%$ bis $31 \%$, während sie in Deutschland um 1,1\%

12 Mittlerweile wurde infolge der Krise das Produktionspotential aller Krisenländer deutlich nach unten revidiert - mit der Folge, dass die strukturellen Defizite nun als deutlich negativ ausgewiesen werden.

13 Sinn, Reutter 2001.

14 Lommatzsch, Tober 2003, 2006. 


\section{Abbildung 4: Nominale Lohnstückkosten \\ 1999 bis 2012, mit durch EZB-Inflationsziel impliziertem Referenz- pfad, $1999=100$}

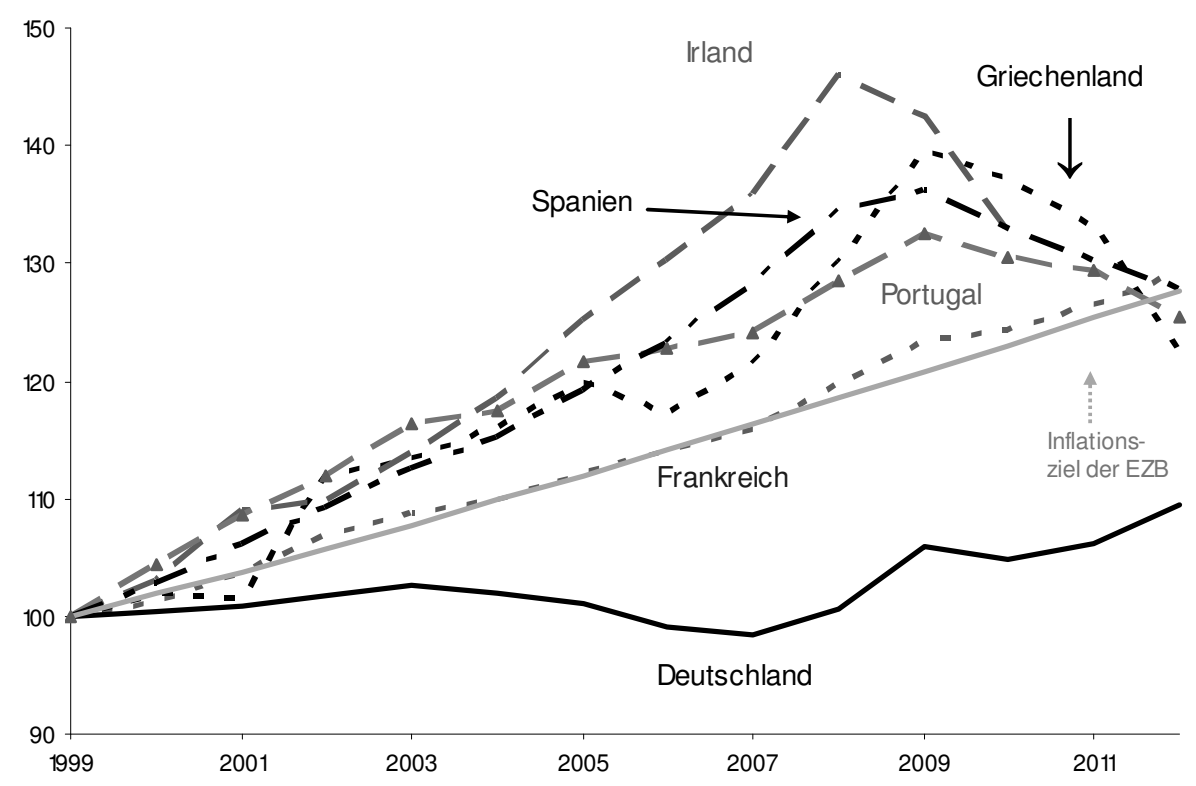

Quelle: AMECO-Datenbank der EU-Kommission (Stand: Mai 2012), eigene Berechnungen.

abnahmen (Abbildung 4). Nicht ganz so ausgeprägt, aber ebenfalls stark unterschiedlich war die Inflationsentwicklung in den einzelnen Ländern. Entsprechend kam es zu deutlichen Verschiebungen in der internationalen preislichen Wettbewerbsfähigkeit der heimischen Unternehmen. Gleichzeitig gab es erhebliche Unterschiede im Realzinsniveau. Während die Realzinsen in den Ländern mit niedriger Inflation und steigender internationaler Wettbewerbsfähigkeit relativ hoch waren und negativ auf die Investitionstätigkeit wirkten, wurde die Binnennachfrage in den Defizitländern infolge relativ hoher Inflationsraten durch relativ niedrige Realzinsen angeregt. In den ersten Jahren der Währungsunion kam der starke Rückgang der Kapitalmarktzinsen in diesen Ländern infolge gesunkener Risikoprämien hinzu. Auf der einen Seite standen somit Länder wie insbesondere Griechenland, Portugal und Spanien mit einer kräftigen Binnenwirtschaft, steigenden Importüberschüssen bzw. zunehmenden Leistungsbilanzdefiziten und auf der anderen Seite vor allem Deutschland mit einer schwachen Binnennachfrage, stark steigenden Nettoexporten und einem zunehmenden Leistungsbilanzüberschuss. Im Jahre 2007 hatten Spanien und Portugal Leistungsbilanzdefizite in Höhe von $10 \%$ des Bruttoinlandsprodukts, Griechenland wies ein Defizit von $17 \%$ auf, während Deutschland und die Niederlande Leistungsbilanzüberschüsse in Höhe 7,5 \% bzw. 8,4 \% verzeichneten. Die 
Folge war eine steigende Auslandsverschuldung in den jetzigen Krisenländern, die in allen Fällen außer Griechenland aber eben nicht eine Folge einer hohen Ausgabefreudigkeit und Verschuldung des Staates selbst, sondern der privaten Unternehmen und Haushalte war.

Länder mit einer einheitlichen Währung und einer einheitlichen Geldpolitik können definitionsgemäß keine unterschiedliche Geldpolitik betreiben. Im Falle von einander abweichender konjunktureller Entwicklungen oder asymmetrisch wirkender Schocks verbleiben aber zwei weitere Makropolitikbereiche, die stabilisierend wirken können: die Fiskalpolitik und die Lohnpolitik. Diese wurden von den Ländern des Euroraums bisher nicht hinreichend eingesetzt. Die Lohn- bzw. Einkommenspolitik erfordert dabei einflussreiche Gewerkschafts- und Unternehmensverbände, die so stark zentralisiert sind, dass sie in der Lage und willens sind, die makroökonomischen Rückwirkungen ihres Handelns mit ins Kalkül zu nehmen. Ist eine "schonende" Einkommenspolitik nicht realisierbar, so muss die Fiskalpolitik im Falle einer überdurchschnittlichen Inflationsentwicklung restriktiv wirken und den Inflationsdruck über eine Dämpfung der wirtschaftlichen Aktivität verringern.

Entsprechend erfordert es die Stabilität in einer Währungsunion, dass ein Land wie Deutschland mit unterdurchschnittlich steigenden Lohnstückkosten und Verbraucherpreisen ihre Binnennachfrage fiskalpolitisch anregt.

\section{Konsolidierung, Wachstum und Abbau der Leistungsbilanzungleichgewichte}

Eine erfolgreiche Strategie der öffentlichen Haushaltssanierung und der Überwindung der Leistungsbilanzungleichgewichte im Euroraum muss so konzipiert sein, dass sie zugleich das Wirtschaftswachstum fördert bzw. nicht übermäßig abbremst. Die Finanzpolitik der Euroländer sollte daher vor dem Hintergrund der eigenen wirtschaftlichen Situation, aber auch mit Blick auf den Euroraum als Ganzes erfolgen. Die Länder mit relativ soliden Finanzen sollten dabei weniger stark konsolidieren, um stabilisierend auf die wirtschaftliche Gesamtentwicklung zu wirken. Angesichts der gegenwärtigen wirtschaftlichen Schwäche sollten sie zudem expansiv wirken, indem sie die Multiplikatorwirkung von öffentlichen Ausgaben nutzen. Diese Wirkung kann auch ohne Kreditfinanzierung deutlich positiv sein, wenn Steuereinnahmen erhöht werden, die die Konjunktur nur wenig schwächen, und zugleich öffentliche Ausgaben erhöht werden, die stark auf die Konjunktur wirken, wie Ausgaben im Bildungssektor - letztere wirken zugleich in Zukunft produktivitätssteigernd. Da die Länder mit relativ soliden Finanzen zugleich jene mit Leistungsbilanzüberschüssen sind, begünstigte eine solche Fiskalpolitik zugleich den Abbau der außenwirtschaftlichen Ungleichgewichte. Dies geschähe über zwei Wege: erstens über das Wachstumsgefälle, das die Importe der Überschussländer relativ stärker ansteigen lässt, und zweitens über das damit einhergehende Inflationsgefälle, das die Wettbewerbsfähigkeit der Defizitländer verbessert.

Alle Länder sollten zudem prüfen, ob zeitlich befristete Erhöhungen der Steuersätze auf hohe Einkommen und gegebenenfalls eine Vermögenssteuer eine relativ zügige Rückführung der in den Jahren 2008 bis 2011 infolge der internationalen 
Wirtschafts- und Finanzkrise entstandenen Zusatzverschuldung - im EuroraumDurchschnitt um 20 Prozentpunkte - ermöglichen kann. ${ }^{15}$ Eine zeitliche Streckung des Konsolidierungskurses erscheint vor dem Hintergrund der schwachen wirtschaftlichen Entwicklung ebenfalls angebracht.

Am allerwichtigsten aber ist es, Maßnahmen zu ergreifen, die das Vertrauen wiederherstellen. Nur wenn die Regierungen signalisieren, dass sie Vertrauen in die Zahlungsfähigkeit aller Euroländer und in die Frühwarnsysteme des Euroraums haben, mit denen künftige Krisen zu vermeiden sind, werden auch Anleger wieder Zuversicht zeigen. Dies hätte zur Folge, dass nicht nur die Zinsen für Staatsanleihen sinken würden, sondern auch jene für Anleihen, die Unternehmen und Banken zahlen müssen. Ersteres erleichtert den Konsolidierungsprozess der Staatshaushalte, letzteres begünstigt zudem die wirtschaftliche Entwicklung direkt. Um ihr eigenes Vertrauen und ihre Entschlossenheit zu zeigen, müssen die Euroländer in irgendeiner Form eine Garantie für die ausstehenden Staatsanleihen geben, beispielsweise indem sie, wie vom Sachverständigenrat vorgeschlagen, einen Schuldentilgungsfonds ins Leben rufen, mit dem alle Staatsschulden, die über 60 \% des BIP hinausgehen, gemeinschaftlich garantiert und einzelstaatlich abgetragen werden. ${ }^{16}$ Sollten sich die Renditen der einzelstaatlich garantierten Staatsschuldtitel dadurch nicht auf niedrigem Niveau angleichen, gäbe der Schuldentilgungsfonds der Europäischen Zentralbank die politische Rückendeckung, die sie benötigt, um zu signalisieren, dass sie mit dem Ziel, die langfristigen Zinsen zu senken, zu unbegrenzten Interventionen am Markt für Staatsanleihen bereit ist. Im günstigsten Fall wäre ein tatsächlicher Einsatz von finanziellen Mitteln gar nicht erforderlich.

Durch die Rückgewinnung des Vertrauens würde die expansive Geldpolitik insbesondere in den Krisenländern stärker als bisher auf die Wirtschaft wirken und damit den restriktiven Impuls der Fiskalpolitik zumindest teilweise kompensieren. Auch würden sich die schiefe Verteilung bei den Refinanzierungsgeschäften der Geschäftsbanken mit der Zentralbank und die TARGET2-Salden zwischen Zentralbanken, die bei der aktuellen Krisenstrategie ein erhebliches Risiko darstellen, ${ }^{17}$ zurückbilden.

\section{Fazit}

Den Regierungen des Euroraums ist es bisher nicht gelungen, die sich seit mehr als zwei Jahren ausbreitende Staatsanleihenkrise zu beenden. Die Europäische Zentralbank trat mehrmals als Retterin in der Not in Aktion. Ohne politische Rückendeckung kann sie aber lediglich einen zeitlichen Spielraum schaffen. Erforderlich ist

15 Nota bene: Die Einhaltung des Defizitkriteriums des Fiskalpakts bleibt davon unbetroffen, da der strukturelle Saldo des Gesamtstaates definiert wird als »der konjunkturbereinigte jährliche Saldo ohne Anrechnung einmaliger oder befristeter Maßnahmen « (Titel III, Artikel 3 (3) a) des Vertrags über Stabilität, Koordinierung und Steuerung in der Wirtschafts- und Währungsunion).

16 Sachverständigenrat 2011, S. 4 ff. und $109 \mathrm{ff}$.

17 Tober 2011.

Leviathan, 40. Jg., 3/2012 
eine Strategie, die die fiskalische Konsolidierung und den Abbau der außenwirtschaftlichen Ungleichgewichte im Euroraum mit Wachstumsimpulsen verbindet.

Ein Schuldentilgungsfonds, der der EZB zudem die Rückendeckung für eine wirksame Stabilisierung der Märkte für Staatsanleihen gäbe, wäre geeignet, das Vertrauen an den Märkten wiederherzustellen. Darüber hinaus muss der Konsolidierungsprozess zeitlich gestreckt und nach Ländern differenziert umgesetzt werden, um das Wachstum im Euroraum zu stützen und zugleich die Leistungsbilanzsalden zu reduzieren. Zur Verringerung der im Zuge der internationalen Wirtschafts- und Finanzkrise stark gestiegenen öffentlichen Schuldenstände wäre eine temporäre Erhöhung von Steuern auf hohe Einkommen und Vermögen sinnvoll. Auch diese Strategie ist mit Risiken verbunden, sie dürften aber deutlich geringer sein als beim gegenwärtigen Vorgehen der Regierungen.

Das oben skizzierte Vorgehen wäre zwar mit dem Fiskalpakt - Kernstück der Krisenstrategie der Regierungen - zu vereinbaren, da Übergangsfristen zu seiner Einhaltung vorgesehen sind. Der Fiskalpakt ist jedoch insbesondere wegen seiner prozyklischen Wirkung und der impliziten Festschreibung einer langfristigen Schuldenstandsquote von rund $30 \%$ zu kritisieren. Er hätte zudem die seit 1999 entstandenen massiven Ungleichgewichte im Euroraum nicht verhindern können. Um eben diese außenwirtschaftlichen Ungleichgewichte abzubauen, setzen die Regierungen - und auch die EZB - derzeit darauf, alle Länder so wettbewerbsfähig zu machen wie Deutschland. Eine hohe internationale Wettbewerbsfähigkeit gibt es aber stets nur in Relation zu Unternehmen aus einem anderen Land. Gewinnen die Länder mit Leistungsbilanzdefiziten und einer hohen Auslandsverschuldung nicht gegenüber den Überschussländern im Euroraum, allen voran Deutschland, an Wettbewerbsfähigkeit, sondern gegenüber dem Rest der Welt, so folgt daraus, dass der Euroraum insgesamt Leistungsbilanzüberschüsse erzielen muss. Und dies wirft die Frage auf, welche Länder außerhalb des Euroraums die entsprechenden Leistungsbilanzdefizite verkraften und später die daraus resultierende Auslandsverschuldung bedienen können.

\section{Literatur}

Buiter, Willem H.; Michels, Jürgen; Rahbari, Ebrahim 2011 a. »TARGETing the wrong villain: TARGET2 and intra-Eurosystem imbalances in credit flows", in Global Economics View, Citigroup Global Markets, June.

Buiter, Willem H.; Michels, Jürgen; Rahbari, Ebrahim 2011 b. The implications of intra-Euro area imbalances in credit flows. Policy Insight 57. London: Centre for Economic Policy Research.

Draghi, Mario 2011. Continuity, consistency and credibility. Introductory remarks by Mario Draghi, President of the ECB, at the 21st Frankfurt European Banking Congress »The Big Shift «, Frankfurt a. M., 18. November 2011.

Europäische Zentralbank 2012. »Konjunkturbereinigung des öffentlichen Finanzierungssaldos «, in Monatsbericht der Europäischen Zentralbank, März 2012, S. 115-119.

Horn, Gustav et al. 2010. Reform des Stabilitäts- und Wachstumspakts: Nicht nur öffentliche, auch private Verschuldung zählt. IMK Report 51. Düsseldorf: Institut für Makroökonomie und Konjunkturforschung, Hans-Böckler-Stiftung. 
Horn, Gustav; Tober, Silke 2007. Wie stark kann die deutsche Wirtschaft wachsen? Zu den Irrungen und Wirrungen der Potenzialberechnung. IMK Report 17. Düsseldorf: Institut für Makroökonomie und Konjunkturforschung, Hans-Böckler-Stiftung.

Lommatzsch, Kirsten; Tober, Silke 2003. »Reform der geldpolitischen Strategie der Europäischen Zentralbank «. in DIW-Wochenbericht 7/2003, S. 110-117.

Lommatzsch, Kirsten; Tober, Silke 2006. »Euro-area inflation - does the Balassa-Samuelson effect matter? ", in International Economics and Economic Policy 3, 2, S. 105-136.

Sachverständigenrat zur Begutachtung der gesamtwirtschaftlichen Entwicklung 2011. Verantwortung für Europa wahrnehmen. Jahresgutachten 2011/12. Wiesbaden.

Sinn, Hans-Werner; Reutter, Michael 2001. The minimum inflation rate for Euroland. NBER Working Paper 8085. Cambridge: National Bureau of Economic Research.

Sinn, Hans-Werner; Wollmershäuser, Timo 2011. Target loans, current account balances and capital flows: the ECB's rescue facility. CESifo Working Paper 3500. München: CESifo.

Storbeck, Olaf 2011. The stealth bailout that doesn't exist: debunking Hans-Werner Sinn. http:// economicsintelligence.com/2011/06/06/the-stealth-bailout-that-doesn \%E2\% 80\%99t-existdebunking-hans-werner-sinn/ (Zugriff vom 13.07.2012).

Tober, Silke 2011. Die Europäische Zentralbank in der Kritik. IMK Report Nr. 67. Düsseldorf: Institut für Makroökonomie und Konjunkturforschung, Hans-Böckler-Stiftung. 
Zusammenfassung: Die EZB trat während der Staatsanleihenkrise mehrfach als Retterin in Aktion. Für Maßnahmen, die das Vertrauen wiederherstellen könnten, fehlt ihr das Mandat. Sie ist bereits Risiken eingegangen, die infolge der inadäquaten Krisenstrategie der Regierungen hoch sind. Die Konsolidierung und der Abbau externer Ungleichgewichte erfordern Zeit und ein differenziertes Vorgehen, das das Wachstum stützt.

Stichworte: Europäische Zentralbank, Staatsanleihenkrise, Fiskalpakt, Leistungsbilanzungleichgewichte

\section{European Central Bank: fighting the crisis alone}

Summary: The ECB repeatedly came to the rescue during the euro-area debt crisis. It lacks, however, the mandate for policy measures sufficient to restore confidence. Because of the inadequacy of the governments' crisis strategy, the ECB has accumulated risks that are substantial. Fiscal consolidation and reducing external imbalances require time and a differentiated approach that allows for growth.

Keywords: European Central Bank, sovereign debt crisis, fiscal compact, current account imbalances

\section{Autorin}

Dr. Silke Tober Institut für Makroökonomie und Konjunkturforschung (IMK)

Hans-Böckler-Stiftung

Hans-Böckler-Straße 39

40476 Düsseldorf

Silke-Tober@boeckler.de 